\title{
DANSS Experiment around the Five Year Milestone.
}

\section{Svirida ${ }^{a, b, 1, *}$}

aAlikhanov Institute for Theoretical and Experimental Physics NRC "Kurchatov Institute",

B. Cheremushkinskaya str. 25, Moscow, 117218, Russia

${ }^{b}$ Lebedev Physical Institute of the Russian Academy of Sciences, Leninskiy avenue 53, Moscow, 119991, Russia

E-mail: Dmitry.Svirida@itep.ru

DANSS detector is composed of plastic scintillator and takes full advantages of its unique location directly below a commercial reactor core. The ability to change the distance to the antineutrino source by means of a lifting platform makes DANSS an ideal apparatus for sterile neutrino searches, while the fine segmentation of the sensitive volume provides an extremely clean separation of the inverse beta decay process. A revised approach to the calibration procedure improved the confidence in the energy scale, while numerous refinements in the Monte-Carlo simulations led to much closer agreement between the experiment and the model. The results of the five year data taking will be presented, including the latest estimates for the exclusion area of the sterile neutrino parameters.

\footnotetext{
*** Particles and Nuclei International Conference - PANIC2021 ***

*** 5 - 10 September, $2021 * * *$

*** Online ***

${ }^{1}$ For the DANSS Collaboration

* Speaker
} 
DANSS is an antineutrino detector composed of 2500 plastic scintillator strips with gadolinium enriched coating forming the sensitive volume of one cubic meter. A lifting gear moves the detector vertically along the axis of a commercial reactor core so that the distance varies from 10.9 to $12.9 \mathrm{~m}$. The reactor body and the building infrastructure above the detector gives $\sim 50 \mathrm{~m}$.w.e. protection from cosmic radiation that stops atmospheric hadrons and adds about 6 times suppression of muons. The multilayer passive and active shielding together with the fine segmentation of the sensitive volume provides an excellent separation of the inverse beta decay (IBD) reaction resulting in better than 50:1 signal to background ratio in the antineutrino detection.

Reaching the neutrino counting rate of about 5000 per day DANSS recorded and analyzed 1.5 million IBD events with (0.75-8) $\mathrm{MeV}$ positrons in addition to the previously reported amount [13]. The total statistics after 5 years of running contains $5.5 \cdot 10^{6}$ antineutrinos in the according energy range and 3 reactor-off periods. New approach to the everyday calibration with cosmic muons that now uses median values instead of MPV's makes the whole procedure more stable. Refined Monte Carlo simulation now includes individual light yields for all photo-detectors which are taken from the actual data. Yet the final energy scale is fixed from the ${ }^{12} \mathrm{~B}$ decays, the processes most similar to the positron ionization signals. Two ways of ${ }^{12} \mathrm{~B}$ production on carbon, either by muons directly or by muon-induced neutrons, can be clearly selected (see fig. 1) and agree within $1 \%$ in terms of the energy scale. Other dedicated processes like emission from radioactive sources and Michel electrons from stopped muon decays are used for further checks and show the agreement with the boron scale within $\pm 1 \%$.

With such an enormous neutrino sample of the cleanest quality many fundamental and applied studies can be performed. The latter include the reactor power measurements, where DANSS demonstrated $1.5 \%$ statistical accuracy for a 2-day exposure in the position closest to the core and only less than $0.5 \%$ systematical bias in 4.5 years of observation. The dependence of the counting rate on the fuel composition seems to be slightly more pronounced than that reported by Daya Bay in [4] and better follows the predictions of the Huber-Mueller (H-M) model [5, 6]. The so called $5 \mathrm{MeV}$ bump is seen by DANSS, but appears twice weaker than should be expected from other experiments if smeared by the DANSS energy resolution. This is also a hint for a better agreement (but not for an exact coincidence) of the experimental spectra with H-M predictions.

The cosmic muons are one of the main sources of the backgrounds in the neutrino detection. Yet their long-term observation leads to splendid byproduct results when correlated to meteorological data. DANSS is capable of muon tracking and thus has perfect muon identification. Left panel of figure 2 shows the muon flux as a function of the calendar time. The seasonal variations should be
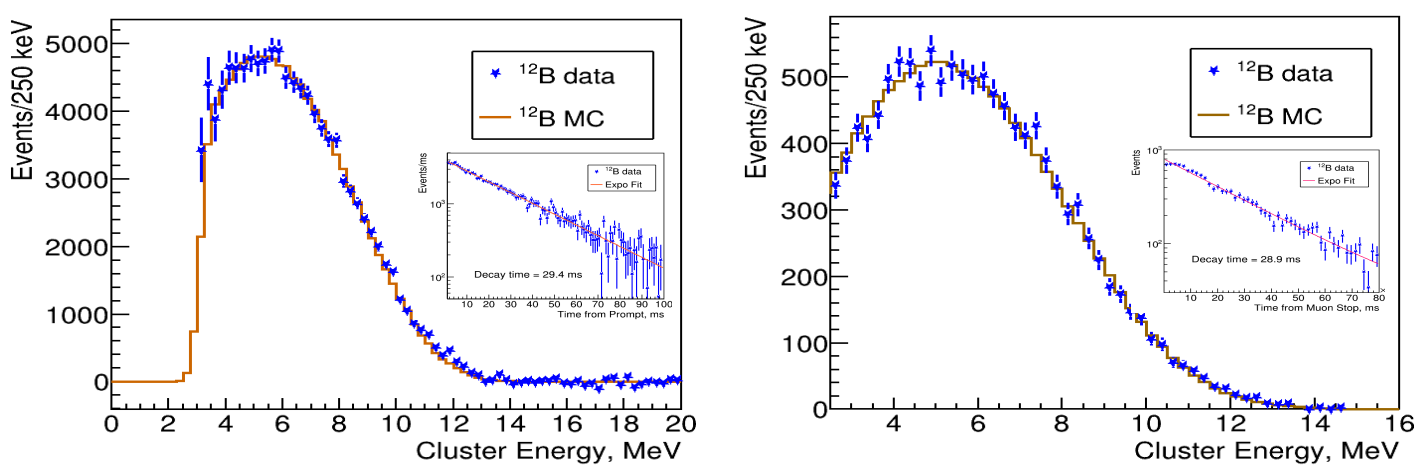

Figure 1: Decay energy spectra of ${ }^{12} \mathrm{~B}$ produced on carbon by fast neutrons (left) and muons (right). The smaller insertions show the exponential fits of the decay time, the values are to be compared to $29.1 \mathrm{~ms}$. 

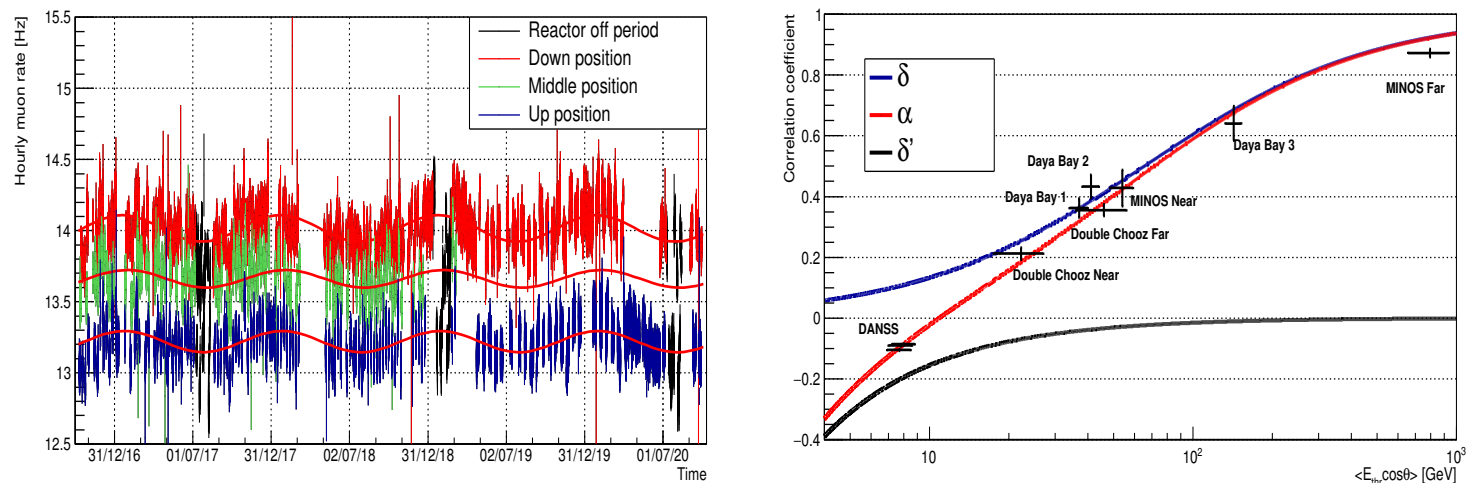

Figure 2: Cosmic muon flux as a function of the calendar time (left) in three detector positions; black points present the reactor-off periods with increased overburden. Right: the temperature correlation coefficient $\alpha$ shown together with the model predictions [7] for its positive $\delta$ and negative $\delta^{\prime}$ components.

attributed to the temperature effect, which is small and negative in case of DANSS - the changes are less than $1 \%$ and have the minimum in summer, on August, 26. The spread of the points around the average sine comes from the barometric effect. The ground pressure is independent on the season, while its hourly jumps are significant. The simultaneous fit of the temperature and pressure data allows the accurate determination of both correlation coefficients. Interestingly, DANSS occupies an intermediate position between the surface and deep underground detectors in terms of the overburden. This is reflected in figure 2 at right where the temperature effect measured by DANSS is compared to other experiments. Mind the good agreement with the model predictions and the sign, opposite to all underground results.

The most fundamental result from DANSS poses stringent limits on the neutrino oscillation parameters to the sterile state by the comparison of the neutrino spectra at different distances from their origin. Many systematic errors are canceled out due to the efficient approach 'various distances - one detector'. The remaining uncertainties in the energy scale and backgrounds are treated as nuisance parameters. No statistically significant evidence for sterile neutrinos was found. The best-fit point for the $4 v$ hypothesis $\Delta m_{14}^{2}=1.3 \mathrm{eV}^{2}, \sin ^{2} 2 \theta_{e e}=0.014$ has a statistical significance of $1.3 \sigma$ only. Based on the Gaussian CLs method, the exclusion region (fig. 3, left) is in a reasonable agreement with the expected sensitivity and reaches $\sin ^{2} 2 \theta_{e e}$ about $8 \cdot 10^{-3}$ in the region of $\Delta m_{14}^{2} \sim 0.9 \mathrm{eV}^{2}$. The best point obtained from the combined fit of the reactor and gallium anomalies [8] is deep in the exclusion region with $\Delta \chi^{2}=107$, which is much more than required for $5 \sigma$ exclusion.

Along with many advantages DANSS has one essential drawback: the energy resolution of the detector has only moderate value of $34 \%$ at $1 \mathrm{MeV}$. An upgrade is planned to eliminate this oversight. New strips with optimized design will have more than twice the light yield and much better spatial light collection homogeneity, which brings the experiment closer to the challenging goal of $13-15 \%$ at $1 \mathrm{MeV}$. In addition the sensitive volume will be extended by $70 \%$. As a result the sensitivity region will be significantly broadened (fig. 3, right) in shorter time, and particularly in the direction of higher sterile neutrino masses. This will give an opportunity to directly check the positive result by Neutrino-4 [9] and will provide a large overlay with allowed areas predicted by several groups, including the recent estimates from BEST [10]. 

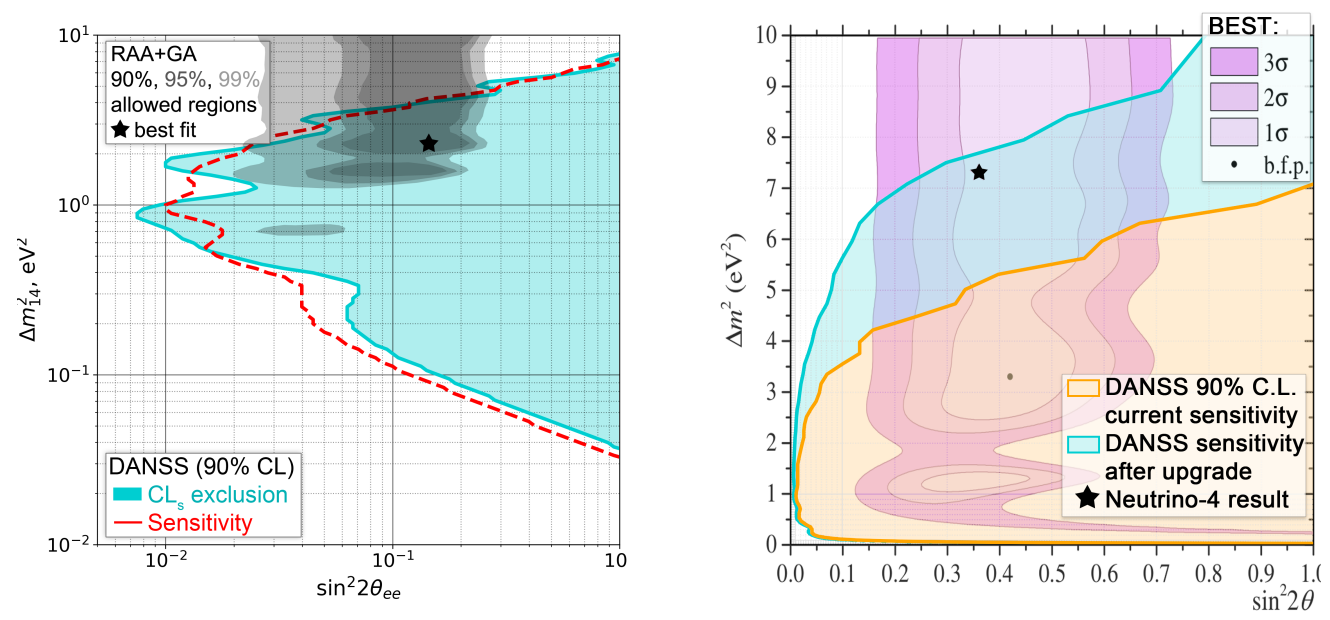

Figure 3: Left: the $4 v$ hypothesis exclusion region and sensitivity from DANSS (preliminary); the star and the gray area shows the best point and the allowed region from the reactor and gallium anomalies [8]. Right: projected sensitivity of upgraded DANSS after 1.5 years of running, compared to the current sensitivity; best points from Neutrino-4 [9] and BEST [10] and the allowed region from the latter are also shown.

\section{Acknowledgments}

DANSS collaboration is grateful to the directorates of ITEP and JINR for incessant support of this work and appreciates the assistance of the KNPP administration and Radiation Safety Department staff. The detector operation and data analysis became possible due to the valuable support from the Russian Science Foundation grant 17-12-01145ח.

\section{References}

[1] M. Danilov for the DANSS Collaboration, New results from the DANSS experiment, PoS ICHEP2020 (2021) 121.

[2] D. Svirida for the DANSS Collaboration, DANSS experiment: current status and future plans, J. Phys. Conf. Ser. 1690 (2020) 012179.

[3] N. Skrobova for the DANSS Collaboration, Statistical data analysis in the DANSS experiment, J. Phys. Conf. Ser. 1690 (2020) 012173.

[4] F.P. An et al., Evolution of the reactor antineutrino flux and spectrum at Daya Bay, Phys. Rev. Lett. 118 (2017) 251801.

[5] P. Huber, Determination of antineutrino spectra from nuclear reactors, Phys. Rev. C84 (2011) 024617.

[6] T.A. Mueller et al., Improved predictions of reactor antineutrino spectra, Phys. Rev. C83 (2011) 054615.

[7] P.H. Barrett et al., Interpretation of cosmic-ray measurements far underground, Rev. Mod. Phys. 24 (1952) 133.

[8] G. Mention et al., The reactor antineutrino anomaly, Phys. Rev. D83 (2011) 073006.

[9] A.P. Serebrov and R.M. Samoilov, Analysis of the Results of the Neutrino-4 Experiment on the Search for the Sterile Neutrino and Comparison with Results of Other Experiments, JETP Lett. 112 (2020) 199, [hep-ex/2003.03199].

[10] V.V. Barinov et al., Results from the Baksan Experiment on Sterile Transitions (BEST), [nucl-ex/2109.11482]. 